\title{
INFLUÊNCIA DA ADIÇÃO DE IODETO DE POTÁSSIO E CITRATO DE SÓDIO NA QUALIDADE DO SÊMEN DE PACU (Piaractus mesopotamicus - Holmberg, 1887)
}

\author{
The effects of potassium iodide and sodium citrate on semen \\ quality of pacu (Piaractus mesopotamicus)
}

\author{
Alexandre Nízio Maria ${ }^{1}$, Luis David Solis Murgas ${ }^{2}$, Márcia Oliveira Barbosa Silva ${ }^{3}$, \\ Alécio B. Miliorini ${ }^{4}$, Renan T. Franciscatto ${ }^{4}$, Priscila Vieira Rosa Logato ${ }^{5}$
}

\section{RESUMO}

Atualmente, tem-se dado maior atenção ao desenvolvimento de tecnologias para estocagem de sêmen de peixes em curto prazo, por dias ou semanas, utilizando diluentes de sêmen que não ativem a motilidade espermática. Essa técnica é indicada em piscicultura para facilitar a reprodução artificial ou aumentar sua eficiência. Conduziu-se este trabalho com o objetivo de verificar o efeito do diluidor BTS (Beltsville Thawing Solution - MINITUB ${ }^{\circledR}$ ) na qualidade do sêmen de pacu, após a coleta, enriquecido com iodeto de potássio e com citrato de sódio. Foram utilizados seis reprodutores de pacu (Piaractus mesopotamicus) submetidos à indução hormonal e liberação dos gametas, manualmente. O sêmen coletado de cada macho foi dividido em três frações, as quais receberam diluição (1:3) com o diluidor 1 (BTS 5\% + iodeto de potássio 0,16\%), diluidor 2 (BTS 5\% + citrato de sódio $1,28 \%$ ) e uma fração não diluída (controle). A ativação espermática foi realizada com água destilada, e, a seguir, foram avaliadas as taxas e duração da motilidade espermática, cujos valores médios respectivos foram de $95,00 \pm 3,16 \%$ e 486,83 $\pm 314,55$ segundos para o sêmen in natura, $80,00 \pm 9,49 \%$ e 221,50 $\pm 207,89$ segundos para o sêmen com o diluidor 1 e $80,00 \pm 11,40 \%$ e $320,66 \pm 181,14$ segundos para o sêmen no diluidor 2 . Em seguida, foram coletadas amostras para a avaliação da concentração espermática, que obteve um valor médio de 13,89 $\pm 1,26 \times 10 \% / \mathrm{mL}$. Pelas análises estatísticas, verificou-se que não houve diferença significativa na duração da motilidade $(\mathrm{P}>0,05)$ entre o sêmen "in natura" e os diluidores 1 e 2 . A motilidade espermática foi estatisticamente melhor $(\mathrm{P}<0,05)$ no sêmen in natura, quando comparada com os diluidores 1 e 2 . Não houve diferença entre os dois diluidores para esse parâmetro. Conclui-se, assim, que os diluidores reduziram a motilidade espermática, sem entretanto, afetar a duração da motilidade, podendo ser utilizado na conservação do sêmen dessa espécie, desde que sua osmolaridade seja aumentada. Há necessidade de mais pesquisas para se avaliar a eficiência desses diluidores várias horas após resfriamento.

Termos para indexação: Diluentes, motilidade espermática, reprodução.

\section{ABSTRACT}

Currently, it has been given a special attention to developing technologies for short-term storage of fish semen, for a few days to weeks, using extenders of semen that do not activate the sperm motility. The short-term storage technique is indicated for fish culture to facilitate the artificial reproduction and to increase its efficiency. The objective of this study was to verify the effect of BTS (Beltsville Thawing Solution - MINITUB ${ }^{\circledR}$ ) as an extender for pacu (Piaractus mesopotamicus) semen. Semen of each of the six males (previously treated with carp pituitary extract) was divided into three fractions: one fraction was kept in natura and the other two fractions were diluited (1:3) with either extender 1 (BTS $5 \%+$ potassium iodide $0,16 \%$ ) or extender 2 (BTS $5 \%+$ sodium citrate $1,28 \%)$. The sperm motility was induced with distilled water, and the percentage and duration of sperm motility was scored. The percentage of moving sperm cell was significantly higher $(\mathrm{P}<0,05)$ in semen in natura $(95,00 \pm 3,16 \%)$ compared with semen diluted in extender $1(80,00 \pm 9,49 \%)$ or $2(80,00 \pm 11,40 \%)$. On the other hand, the duration of sperm motility was not affected by any extender $(\mathrm{P}>0,05)$ and ranged from $221,50 \pm 207,89$ seconds (semen diluted in extender 1 ) to $486,83 \pm 314,55$ seconds (semen in natura). After that samples for the evaluation of the sperm concentration were collected, with an average value of about $13,89 \pm 1,26 \times 10^{9} / \mathrm{mL}$. One can conclude, that BTS may be used to preserve pacu semen, as long as the salts used may be added on a higher concentration, in order to increase the BTS solution osmolarity. It is also suggested that other salts should be tested.

Index terms: Extenders, sperm motility, reproduction.

(Recebido para publicação em 13 de janeiro de 2003 e aprovado em 15 de abril de 2003)

\footnotetext{
1. Zootecnista, mestrando em Zootecnia (Aquicultura) no Departamento de Zootecnia da Universidade Federal de Lavras/UFLA - Caixa Postal 37 - $37200-000$ - Lavras, MG.

2. Professor Adjunto do Departamento de Medicina Veterinária/UFLA.

3. Bióloga - doutoranda em Zootecnia DZO/UFLA

4. Acadêmicos do curso de Medicina Veterinária/UFLA

5. Professora Adjunto do Departamento de Zootecnia/UFLA.
} 


\section{INTRODUÇÃO}

O pacu (Piaractus mesopotamicus) é um peixe de grande porte que vem sendo utilizado nas pisciculturas e nos repovoamentos de reservatórios. A criação dessa espécie tem sido incentivada em razão do seu bom valor comercial e da grande aceitação no mercado nacional (VAZ et al., 2000), juntamente com a questão ambiental, visando à redução de impactos gerados pelo represamento dos rios.

Os peixes de piracema, que constituem parte significativa das espécies comerciais brasileiras, não se adaptam ao regime de águas lênticas dos reservatórios. Essas espécies de peixes necessitam da dinâmica da correnteza fluvial para o desenvolvimento cíclico de seus órgãos sexuais, maturação dos gametas e estímulo para a reprodução (GODOY, 1975).

Em cativeiro, embora essa espécie se adapte com grande facilidade, não se reproduz, a menos que seja submetida à indução hormonal. Sob essas condições, o período reprodutivo ocorre do final de novembro até o início de janeiro (ROMAGOSA et al., 1998).

Poucos trabalhos têm obtido sucesso na conservação da qualidade do sêmen de pacu em curto prazo, utilizando a redução da temperatura. Essa técnica é relativamente simples, não requerendo equipamentos sofisticados e pode levar benefícios técnicos e econômicos às estações de piscicultura (HARVEY e KELLEY, 1988). Segundo Franciscatto et al. (2002), a conservação seminal em curto período de tempo permite que o sêmen esteja apto para a fecundação de ovócitos sem a presença do macho, melhorando a eficiência das reproduções em cativeiro.

Os espermatozóides dos peixes são imóveis nas gônadas e somente apresentam motilidade quando do contato com a água ou outro meio suficientemente hiposmótico. Portanto, na preservação espermática, é necessário primar por um meio de osmolaridade capaz de assegurar a imobilidade das células espermáticas, a fim de se resguardarem as reservas enérgicas, principalmente. Ihering e Azevedo (1935) mostraram que em peixes neotropicais a redução da osmolaridade do meio diluente é acompanhada pela iniciação da motilidade espermática.

Grandes esforços foram feitos para desenvolver diluentes complexos que imitassem a composição do plasma seminal. Todavia, as únicas condições exigidas de um diluente são que ele seja carreador de crioprotetores e que não ative a motilidade espermática (HARVEY e CAROSFELD, 1993).
Cada espécie requer seu próprio diluente (OTT e HORTON, 1971), embora alguns diluentes desenvolvidos para espécies em particular possam ser usados com sucesso em outras espécies (LEGENDRE e BILLARD, 1980). O diluidor BTS (Beltsville Thawing Solution MINITUB $^{\circledR}$ ) tem sido muito utilizado na rotina de resfriamento do sêmen suíno. Contudo, bons resultados são encontrados com sêmen de algumas espécies de peixes (MILIORINI et al., 2002; MURGAS et al., 2002; FRANCISCATTO et al., 2002). Sendo assim, objetivou-se adicionar ao diluidor BTS alguns solutos, tais que, além de fornecerem osmolaridade ideal para a conservação dos espermatozóides imóveis, pudessem contribuir para a integridade da célula espermática, verificando sua influencia na taxa e duração da motilidade espermática no sêmen de pacu, imediatamente após sua coleta.

\section{MATERIAL E MÉTODOS}

O presente experimento foi realizado durante a piracema do ano 2001/2002, conduzido inicialmente na Estação de Piscicultura da Usina Hidrelétrica da Companhia Energética de Minas Gerais (CEMIG), em Itutinga, MG, onde se efetuaram a coleta e a avaliação do sêmen. A concentração espermática foi realizada no Laboratório de Fisiologia e Farmacologia do Departamento de Medicina Veterinária da Universidade Federal de Lavras (UFLA), Lavras, MG.

Foram utilizados seis reprodutores de pacu (Piaractus mesopotamicus) provenientes dos tanques de criação da estação de piscicultura, que apresentaram um peso corporal médio de 3,6 $\pm 0,19 \mathrm{~kg}$, submetidos a tratamento hormonal com extrato bruto de hipófise de carpa (EBHC), nas dosagens de 0,3 e 3,0 mg de $\mathrm{EBHC} / \mathrm{kg}$ de peso corporal (prévia e efetiva, respectivamente), segundo a rotina utilizada na estação. Após 24 horas da aplicação da dosagem de EBHC efetiva, utilizando um puçá, retirou-se do tanque de tratamento um macho de cada vez. Para imobilização do animal, utilizouse toalha umedecida, sendo a região circunvizinha ao poro urogenital seca e limpa, a fim de se prevenir uma ativação e/ou contaminação das amostras por fezes ou urina.

O critério de seleção dos reprodutores baseou-se no rubor apresentado pela papila urogenital e na liberação de sêmen, mediante leve pressão, no sentido crânio-caudal, aplicada à parede celomática. Foram observadas inicialmente a coloração e a viscosidade do sêmen coletado, para se evitar o uso de amostras eventualmente contaminadas por urina, fezes, sangue ou água. 
O sêmen foi recolhido em tubos de ensaio graduados e as amostras foram divididas em três frações de mesmo volume. Duas frações receberam diluição na proporção de 1:3 (sêmen:diluidor) e uma fração não foi diluída (controle). O diluidor testado foi o BTS $5 \%$ (Beltsville Thawing Solution - MINITUB ${ }^{\circledR}$ ) enriquecido com iodeto de potássio $0,16 \%$ (diluidor 1 ) e o BTS $5 \%$ enriquecido com citrato de sódio $1,28 \%$ (diluidor 2).

Na tabela 1 encontra-se a composição do diluidor BTS contida em 100 gramas do produto.

TABELA 1 - Composição em 100 gramas de BTS MINITUB $^{\circledR}$.

\begin{tabular}{|c|c|}
\hline 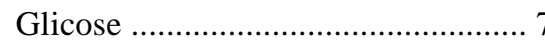 & $78,40 \mathrm{~g}$ \\
\hline 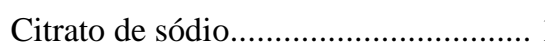 & $12,71 \mathrm{~g}$ \\
\hline 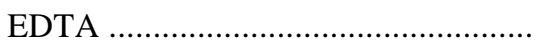 & $2,65 \mathrm{~g}$ \\
\hline Bicabornato de sódio .............................. & $2,65 \mathrm{~g}$ \\
\hline 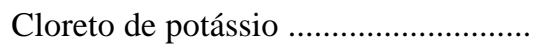 & $1,59 \mathrm{~g}$ \\
\hline Sulfato de neomicina .......................... & $2,00 \mathrm{~g}$ \\
\hline
\end{tabular}

As determinações da taxa de motilidade e duração da motilidade foram feitas em microscópio óptico após gotejamento de cerca de $2 \mu \mathrm{L}$ de sêmen sobre uma lâmina de vidro previamente focalizada em 100 X. A caracterização da motilidade espermática foi baseada na taxa de espermatozóides móveis, utilizando uma escala arbitrária de 0 a $100 \%$, estimada por um único observador. $\mathrm{O}$ sêmen in natura e o diluído foram ativados com água destilada. A partir desse momento, o cronômetro foi ativado para registrar a duração da motilidade do espermatozóide até atingir a taxa de $10 \%$ de motilidade (MILIORINI et al., 2002; MURGAS et al., 2002).

A concentração espermática foi estimada com o auxilio de uma câmara hematimétrica. Um volume de $0,001 \mathrm{~mL}$ de sêmen foi diluído em $1 \mathrm{~mL}$ de solução de formol citrato (citrato de sódio 2,9 gramas, água destilada $100 \mathrm{~mL}$, solução comercial formaldeido 35\%). Uma alíquota dessa solução foi retirada e colocada na câmara hematimétrica, onde o número de espermatozóides por $\mathrm{mL}$ foi obtido (MILIORINI et al., 2002; MURGAS et al., 2002).

Utilizou-se um delineamento em blocos ao acaso, com seis blocos (um animal por bloco). Os resultados foram submetidos à análise estatística pelo Sistema de Análise de Variância (FERREIRA, 1999), sendo as médias de motilidade e duração da motilidade comparadas pelo teste de Scheffé a 5\% de probabilidade.

\section{RESULTADOS E DISCUSSÃO}

A concentração espermática observada no sêmen de pacu foi de $13,89 \pm 1,26 \times 10^{9}$ espermatozóides/mL. Esses resultados diferem dos obtidos para a mesma espécie, tanto por Silveira et al. (1990), com médias de concentração de 28,07 $\pm 8,2 \times 10^{9}$ espermatozóides $/ \mathrm{mL}$ quanto por Miliorini et al. (2002), cuja média foi de $18,62 \pm 3,31 \times 10^{9}$ espermatozóides/mL. Essa variação dos resultados pode estar relacionada com a época do ciclo reprodutivo na qual a coleta do sêmen foi realizada.

Os valores médios da taxa de motilidade espermática e sua duração para o sêmen in natura foram de $95,00 \pm 3,16 \%$ e $486,83 \pm 314,55$ segundos. Miliorini et al. (2002), trabalhando com a mesma espécie, observaram valores de $99,16 \pm 0,83 \%$ de motilidade espermática com média de $62,50 \pm 3,48$ segundos de duração para o sêmen in natura.

Após a diluição do sêmen com os diluidores 1 e 2, a taxa de motilidade espermática reduziu-se significativamente $(\mathrm{P}<0,05)$ a $80,00 \pm 9,49 \%$ com o diluidor 1 e a $80,00 \pm 11,40 \%$ com o diluidor 2 . Miliorini et al. (2002), após testarem uma solução diluidora de sêmen contendo BTS, cloreto de potássio e o crioprotetor dimetilsulfóxido, constataram que a média da taxa de motilidade espermática reduziu-se a $90 \pm$ $1,07 \%$, imediatamente após sua diluição. Os dois diluidores nesse parâmetro apresentaram valores semelhantes, com 80,00\% de média de motilidade (Tabela 2).

A variável duração da motilidade espermática não apresentou diferença significativa entre o sêmen in natura e o diluído em meio BTS $5 \%$ enriquecido $(\mathrm{P}>0,05)$.

Na Tabela 2, estão apresentadas as taxas e a duração da motilidade espermática média das amostras de sêmen in natura e diluído em meio BTS enriquecido.

Com os resultados encontrados, conclui-se que os diluidores reduziram a motilidade espermática sem afetar significativamente a duração da motilidade. O diluidor BTS apresenta um potencial de utilização na conservação do sêmen dessa espécie, desde que sua osmolaridade seja aumentada. No entanto, necessita-se de mais pesquisas para se avaliar a eficiência desses diluidores várias horas após o resfriamento. 
TABELA 2 - Taxa e duração da motilidade espermática do sêmen de pacu in natura e diluído em meio BTS $5 \%$ enriquecido com iodeto de potássio e citrato de sódio (Média \pm D.P.).

\begin{tabular}{lccc}
\hline \multirow{2}{*}{ Parâmetros } & $\begin{array}{c}\text { Sêmen } \\
\text { in natura }\end{array}$ & \multicolumn{2}{c}{ Diluidor BTS } \\
\cline { 3 - 4 } & & $\begin{array}{c}\text { Iodeto de } \\
\text { potássio }\end{array}$ & $\begin{array}{c}\text { Citrato de } \\
\text { sódio }\end{array}$ \\
\hline Motilidade & $95,00 \pm$ & $80,00 \pm$ & $80,00 \pm$ \\
$(\%)$ & $3,16 \mathrm{a}$ & $9,49 \mathrm{~b}$ & $11,40 \mathrm{~b}$ \\
Duração da & $486,83 \pm$ & $221,50 \pm$ & $320,66 \pm$ \\
motilidade & $314,55 \mathrm{a}$ & $207,89 \mathrm{a}$ & $181,14 \mathrm{a}$ \\
(segundos) & & & \\
\hline
\end{tabular}

Médias seguidas da mesma letra nas linhas não diferenciam entre si pelo teste Scheffé $(\mathbf{P}>0,05)$.

\section{AGRADECIMENTOS}

À Companhia Energética de Minas Gerais CEMIG (Estação Ambiental de Itutinga), pelo auxilio prestado na realização deste trabalho.

\section{REFERÊNCIAS BIBLIOGRÁFICAS}

FERREIRA, D. F. Sistema de análise de variância SISVAR. Ver. 4.3. Lavras: UFLA-Departamento de Ciências Exatas, 1999.

FRANCISCATTO, R. T.; MURGAS, L. D. S.; MILIORINI, A. B.; SILVA, M. O. B.; LOGATO, P. V. R. Qualidade do sêmen de curimba (Prochilodus lineatus) e taxa de fertilidade após resfriamento a $4^{\circ} \mathrm{C}$. Revista Brasileira de Reprodução Animal, Belo Horizonte, v. 26, n. 3, p. 213-215, jul./set. 2002.

GODOY, M. P. Peixes do Brasil. Piracicaba: Franciscana, $1975.4 \mathrm{v}$.

HARVEY, B.; CAROSFELD, J. Induced breeding in tropical fish culture. Otawa: International Development Research Center, 1993. 144 p.

HARVEY, B. J.; KELLEY, R. N. Pratical methods for chilled and frozen storage of tilapia spermatozoa. In: PULLING, R. R. V.; BHUKASWAN, T.; TONGATHAI, K.; MACLEAN, J. L. (Eds.). INTERNATIONAL SYMPOSIUM ON TILAPIA IN AQUACUlTURE, 2., 1988. Procedings... [S.1.]: ICLARM, 1988. p. 15.
IHERING, R. V.; AZEVEDO, P. Experiências com o esperma da curimatã (Prochilodus) dos açudes salgados da Paraíba. Annual Acad. Bras. Sciencias, [S.1.], v. 7, p. 19-27, 1935.

LEGENDRE, M.; BILLARD, R. Cryopreservation of rainbow trout sperm by deep-freezing. Reproduction Nutrition Development, [S.1.], v. 20, p. 1859-1868, 1980.

MILIORINI, A. B.; MURGAS, L. D. S.; VIVEIROS, A. T. M.; FRANCISCATTO, R. T.; SILVA, M. O. B.; MARIA, A. N. Resfriamento do sêmen de pacu (Piaractus mesopotamicus) à $4^{\circ} \mathrm{C}$, utilizando diferentes concentrações de dimetilsulfóxido. Revista Brasileira de Reprodução Animal, Belo Horizonte, v. 26, n. 3, p. 209-211, jul./set. 2002.

MURGAS, L. D. S.; MILIORINI, A. B.; SILVA, M. O. B.; FRANCISCATTO, R. T.; MARIA, A. N. Viabilidade seminal de piapara (Leporinus obtusidens), empregando-se diferentes diluentes, no resfriamento do sêmen à $4^{\circ} \mathrm{C}$. Revista Brasileira de Reprodução Animal, Belo Horizonte, v. 26, n. 3, p. 211-213, jul./set. 2002.

OTT, A. G.; HORTON, H. F. Fertilization of chinook and coho salmon eggs with cryopreserved sperm. Journal Fish Research Bd. Canadian, [S.1.], v. 28, p. 745-748, 1971.

ROMAGOSA, E.; PAIVA, P. de; GODINHO, H. M.; STORFER, R. B. Desenvolvimento dos ovócitos de Piaractus mesopotamicus (Holmberg, 1887) (=Colossoma mitrei, 1985) em condições de cultivo intensivo. Ciência e Cultura, Lavras, v. 40, n. 1, p. 60-64, 1998.

SILVEIRA, W. F. da; KAVAMOTO, E. T.; CESTAROLLI, M. A.; GODINHO, H. M.; RAMOS, S. M.; SILVEIRA, A. N. Avaliação espermática, preservação criogênica e fertilidade do sêmen do pacu, Piaractus mesopotamicus (Holmberg, 1887), proveniente de reprodução induzida. Boletim do Instituto de Pesca, São Paulo, v. 17, p. 1-13, 1990.

VAZ, M. M.; TORQUATO, V. C.; BARBOSA, N. D. de C. Guia ilustrado de peixes da bacia do rio Grande. Belo Horizonte: CEMIG/CETEC, 2000. $144 \mathrm{p}$. 